\title{
Sinergie, Italian Journal of Management
}

\section{Cari Lettori,}

come preannunciato nell'editoriale del numero 93 e ribadito in occasione del Convegno di Cassino del novembre scorso, eccoci ad adottare da questo numero 95 il nuovo nome della rivista.

Già nell'editoriale richiamato abbiamo illustrato il cammino culturale che ci ha portato a questo cambiamento, accogliendo il suggerimento della comunità scientifica di Sinergie che ci invitava ad accelerare il processo di internazionalizzazione, senza peraltro rinunciare alla sua identità nazionale.

Da qui la scelta del nuovo titolo, che riflette l'italianità nel mantenimento del nome Sinergie nella nostra lingua, agganciandolo nel contempo alla specificazione dell'oggetto di studio espresso in lingua inglese.

Il cambiamento sotteso a questa scelta non intende essere solo di facciata. Sappiamo bene che non basta cambiare il titolo di una rivista per elevarne l'apertura internazionale.

Con la gradualità del caso, Sinergie passerà così a una impostazione integralmente fondata sull'uso della lingua inglese, riconoscendone l'attuale valore di codice linguistico universale della ricerca scientifica.

In questa prospettiva, peraltro, già si muove il primo numero del 2015 frutto del Call for paper per lo Special issue on Sustainability, Stakeholder and Business.

L'obiettivo che ci siamo posti con questa scelta è di portare la Rivista con la sua indipendenza culturale e con i suoi contenuti al di là dei confini nazionali.

Questo almeno per due ragioni. Da una parte, per contribuire alla diffusione del pensiero manageriale italiano oltre i confini del Paese. Dall'altra, per creare uno spazio capace di attrarre un dibattito internazionale che sappia interpretare e, perché no, anticipare, i cambiamenti nella condotta delle imprese.

In questo senso il Journal è aperto a contributi dai quali emergano scenari e visioni del futuro che possano contribuire a rileggere in forma critica il divenire dell'impresa, nell'ambito del susseguirsi dei cambiamenti culturali e socioeconomici con i quali il management deve continuamente misurarsi.

Così, come puntualizzato nelle politiche editoriali della rivista, Sinergie si propone di promuovere contributi sia empirici che concettuali che non siano meramente descrittivi e/o quantitativi, bilanciando la relevance con il rigor e favorendo l'interpretazione, la discussione critica e il ragionamento, rispetto alla pura misurazione di fenomeni più o meno significativi.

Va precisato, inoltre, che la rivista non intende caratterizzarsi unicamente come uno spazio specialistico destinato a parlare solo alla comunità scientifica. Questa è, e sarà sempre, il nostro primo riferimento entro il quale confrontare le conoscenze che 
saremo capaci di produrre. Ma sarà anche nostro compito valutare come assolvere ad un ruolo di propulsione, impulso, stimolo, spinta, apertura ad idee a sostegno dell'efficacia dell'azione manageriale sul campo.

In questo senso, Sinergie si presenta come uno spazio dedicato alla produzione e alla diffusione di conoscenze con il duplice intento di contribuire al progresso della riflessione scientifica e di formulare proposte di valore alle quali il management possa accedere.

Sinergie, in altri termini, si prefigge di costruire un ponte tra la ricerca e il mondo delle imprese per agevolare la comprensione reciproca del divenire di una complessità che, seppur tipica del dinamismo ambientale, rivela negli ultimi tempi intensità e livelli inusuali, tali da porre in discussione i paradigmi che sostengono il sistema di mercato nelle forme conosciute.

Per agevolare il conseguimento di questi obiettivi anche l'editing della Rivista, come pure il suo portale web, cambieranno, l'uno dopo l'altro, nella direzione di una sua più agevole lettura e della capacità di ospitare tutte le innovazioni dirette a fare di Sinergie, Italian Journal of Management un sempre più utile strumento di lavoro per gli studiosi e gli operatori di management italiani e internazionali.

Nell'incamminarci con entusiasmo in questa nuova avventura, cogliamo l'occasione per ringraziare voi Lettori, gli Autori dei lavori pubblicati e di quelli per qualche motivo non accolti, il Comitato Scientifico, i Referee e la Redazione per aver consentito con azioni, idee e partecipazione di creare il valore culturale che Sinergie esprime. 